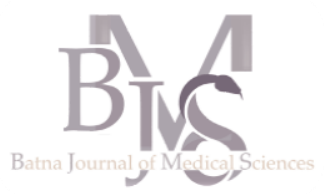

Recherche des anticorps antiplaquettaires par cytométrie en flux, chez les patients présentant un purpura thrombopénique immunologique.

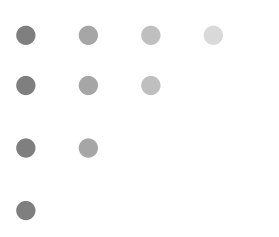

${ }^{1}$ Laboratoire central, Hôpital Militaire Régional Universitaire de Constantine - Algérie.

2 Faculté de Médecine,

Université Constantine 3 Salah Boubnider - Algérie.

${ }^{3}$ Service d'hématologie et de thérapie cellulaire, EHU $1^{\circ}$ novembre 1954, Oran - Algérie. ${ }^{4}$ Faculté de Médecine, Université Oran 1 Ahmed Ben Bella - Algérie.

\section{Correspondance à :}

Mohamed BRAHIMI

dr.brahimi.m@gmail.com

DOI :https://doi.org/10.48087/ BJMSra.2021.8113

\section{Historique de l'article :}

Reçu le 13 décembre 2020

Accepté le 23 mai 2021

Publié le 04 juin 2021

Il s'agit d'un article en libre accès distribué selon les termes de la licence Creative Commons Attribution International License (CC BY 4.0), qui autorise une utilisation, une distribution et une reproduction sans restriction sur tout support ou format, à condition que l'auteur original et la revue soient dûment crédités.

Pour citer l'article :

Zouitene R et Brahimi M. Recherche des anticorps antiplaquettaires par cytométrie en flux, chez les patients présentant un purpura thrombopénique immunologique. Batna J Med Sci 2021;8(1):72-6. https://doi.org/10.48087/B ذMSra.2021.8113

\title{
Detection of anti-platelet antibodies by flow cytometry in patients with immunological thrombocytopenic purpura.
}

\author{
Raouf Zouitene ${ }^{1,2}$, Mohamed Brahimi ${ }^{3,4}$.
}

\section{RÉSUMÉ}

En raison de la non disponibilité d'un test biologique qui confirme le diagnostic du PTI « « purpura thrombopénique immunologique », ce dernier se pose par élimination de toutes les autres causes secondaires d'une thrombopénie. La cytomètrie en flux utilisant la fluorescence, a permis la détection des immunoglobulines associées aux membranes plaquettaires appelées PAIG (Platelet associated immunoglobulin). La recherche des PAIG représente une technique très prometteuse qui peut être appliquée pour le dépistage du PTI selon les nombreuses études réalisées dans ce sujet. D’autres versions modifiées de la recherche des PAIG ont permis une quantification des PAIG, en utilisant une calibration à base de microbilles fluorescentes ou des plaquettes témoins positives sensibilisées par des Ig de surface. L'utilisation des microbilles dans les études récentes a permis l'identification des glycoprotéines plaquettaires, cibles des autoanticorps, la technique est appelée FCIA (flow cytometry immunobead assay). Le but de cette revue de littérature est de rapporter les principes et les résultats de ces techniques.
\end{abstract}

Mots Clés : Purpura Thrombopénique Immunologique ; cyrtométrie en flux ; anticorps

\section{INTRODUCTION}

Selon les directives de la société américaine d'hématologie (ASH) de 2011 [1], le purpura thrombopénique immunologique (PTI) est un désordre auto-immun caractérisé par la destruction immunologique des plaquettes circulantes, sous un stimulus inconnu. Le PTI peut se présenter sous une forme isolée (PTI primaire) ou associée à d'autres pathologies (PTI secondaire). Ces causes secondaires incluent, les désordres auto-immuns (particulièrement le syndrome des antiphospholipides), les infections virales (HCV, HIV), et certaines prises médicamenteuses. Son incidence annuelle est de 3 à 4 cas $/ 100000$, avec une prévalence d'environ 50 cas / $10^{5}$ habitants, selon des études britanniques [2].

Le PTI primaire est défini par un taux de plaquettes inférieur à $100 \mathrm{G} / \mathrm{L}$, en absence de toutes autres causes d'une thrombopénie. Sa physiopathologie est comparable à celle de l'anémie hémolytique auto-immune AHAI, où les cellules sensibilisées par des anticorps à leurs surfaces, vont être détruites dans le système réticulo-endothéliale [3]. Outre la destruction périphérique des plaquettes, la synthèse médullaire peut être aussi insuffisante ou inadéquate, responsable d'une aggravation de la chute de la numération plaquettaire [3].

\section{ABSTRACT}

Due to the unavailability of a biological test that confirms the diagnosis of ITP, the latter is done by elimination of all other secondary causes of thrombocytopenia. Flow cytometry using fluorescence, allowed the detection of immunoglobulins associated with platelet membranes called PAIG (Platelet associated immunoglobulin). The PAIG testing represent a very promising technique that can be applied for the screening of the ITP according to many studies carried out in this subject. Other modified versions of PAIG research have allowed the quantification of PAIG, using fluorescent microbead calibration or positive control platelets sensitized with surface Ig. The use of microbeads in recent studies has enabled the identification of platelet GPs which are the targets of the autoantibodies, the technique is called FCIA (flow cytometry immunobead assay). The aim of this literature review is to report the principles and the results of these techniques.

Key words: Immune Thrombopenic Purpura; flow cytometry; antiplatelet antibodies; PAIG; FCIA.

Les auto-anticorps antiplaquettaires sont souvent des IgG dirigés contre le complexe glycoprotéique (GP) GPIIb/IIIa, et/ou le complexe GPIb/IX [3-6].

En raison de la non disponibilité d'un test biologique qui confirme le diagnostic du PTI, ce dernier se pose par élimination de toutes les autres causes secondaires d'une thrombopénie [1-7]. Il est basé sur: L'historique de la pathologie [7], l'examen clinique, ainsi que l'examen de l'hémogramme et du frottis sanguin.

L'optimisation d'un test biologique explorant le PTI, a fait l'objet de nombreuses études où les auteurs ont cherché une éventuelle relation entre le PTI et: les anticorps antiphospholipides et anti-nucléaires, le dosage de thrombopoïétine ou bien les paramètres plaquettaires mesurés par les analyseurs d'hématologie. Cependant tous ces tests sont considérés comme insuffisants par l'ASH) pour la mise en routine dans le diagnostic du PTI, selon les dernières directives de 2011[1].

Néanmoins parmi ces tests, la recherche des anticorps antiplaquettaires fournie des résultats de plus en plus satisfaisants, et commence à être utilisée en routine par plusieurs laboratoires. 
La cytométrie de flux (CMF), a émergée à partir des années 70 s, comme un outil très puissant dans l'étude des antigènes de membranes cellulaires, et intra-cytoplasmiques par l'utilisation des anticorps couplés à des fluorochromes. C'est une technique rapide, performante, très objective et ne requière qu'un petit volume d'échantillon, elle est également réalisable même pour les prélèvements pauvres en cellules. La CMF a donc éliminé les problèmes relatifs à la radioactivité des méthodes radio immnuno assay (RIA), et à la richesse plaquettaire de la monoclonal antibody immobilization of platelet antigens (MAIPA).

La CMF utilisant la fluorescence, a permis la détection des immunoglobulines associées aux membranes plaquettaires appelées Platelet associated immunoglobulin (PAIG). Une technique très prometteuse qui peut être appliquée pour le dépistage du PTI, selon les nombreuses études réalisées dans ce sujet. D'autres versions modifiées de la recherche des PAIG ont permis une quantification des PAIG, en utilisant une calibration à base de microbilles fluorescentes ou des plaquettes témoins positives sensibilisées par des Ig de surface.

L'utilisation des microbilles dans les études récentes a permis l'identification des GP plaquettaires, cibles des auto-anticorps, la technique est appelée flow cytometry immunobead assay (FCIA).

\section{RECHERCHE DES AUTOANTICORPS PLAQUETTAIRES (PAIG)}

La recherche des PAIG par cytométrie en flux, est une technique rapide, très simple et moins couteuse par rapport aux autres techniques. Elle offre également la possibilité d'analyse même dans le cas des thrombopénies sévères, une situation très fréquente dans le cas des PTI surtout en phase aigüe, ce taux de plaquettes très bas représente la principale limite des techniques les plus spécifiques, MAIPA et ELISA.

En raison de sa facilité et rapidité, elle a fait l'objet de nombreuse publications et modifications, depuis les années 70 jusqu'à nos jours, elle consiste à l'utilisation d'un anticorps anti-immunoglobuline humaine fluorescent dirigé contre une suspension de plaquettes lavées du patient PTI pour le test direct. Pour le test indirect, le sérum du patient est incubé avec des plaquettes normales, et puis la révélation des PAIG sérique se fait par une anti globuline humaine fluorescente [6, 8-16]. L'anticorps (Ac) le plus fréquemment utilisé est le Goat anti-human imunoglobin (GAH), couplés à l'Isothiocyanate de fluorescéine (FITC), seul ou en association avec GAH IgM.

Bien que la majorité des PAIG sont d'isotype IgG seul ou en association avec IgM ou IgA $[\mathbf{9 , 1 0 , 1 3 ] , d e s ~ f o r m e s ~} \operatorname{Ig} M$ seule et plus rarement IgA seule ont été rapportés. Rosenfeld [13] a démontré que sur 44 patients PTI, 16\% étaient positif IgM uniquement, la sensibilité du test en utilisant l'IgG seul était de $75 \%$, tandis qu'en associant la recherche d'IgM avec l'IgG la sensibilité atteint 90,9\%.

L'association anti-IgG-anti-IgM-anti-IgA permet le dépistage des formes IgM ou IgA isolées. En outre il semble que chez les enfants, le taux des PAIG de type IgM est plus élevé que l'IgG, néanmoins l'isotype de l'anticorps (IgG ou IgM) n'influence pas la présentation clinique de la pathologie [6].

L'utilisation des anticorps monoclonaux anti-globulines humaines, peut détecter seulement $60 \%$ des IgG présent à la surface des plaquettes, l'utilisation d'une anti IgG polyclonale, donne un signale plus puissant, par détection d'un nombre plus élevé des Ig de surface [10], ceci s'explique par le fait que les anticorps monoclonaux reconnaissent un seul épitope sur la molécule IgG, cet épitope n'est pas toujours accessible pour
l'Ac monoclonal. Les anticorps polyclonaux sont plus utiles et plus pratique, car ils reconnaissent plusieurs épitopes sur l'immunoglobuline.

Les résultats de cette technique, sont exprimés soit en intensité moyenne de fluorescence (MFI) de la population plaquettaire $[6,10,11,15,16]$, ou bien en pourcentage des plaquettes positives $[\mathbf{8}, \mathbf{9}, \mathbf{1 3}, \mathbf{1 4}]$. Cependant on note une grande divergence des résultats obtenus par les différents auteurs (tableau1), ceci est en rapport avec le nombre d'échantillons analysés, les anticorps utilisés, le mode de préparation de l'échantillon.

La méthode d'analyse des résultats et la détermination du seuil de positivité appliquée et les critères diagnostiques utilisés par chaque auteur. Une bonne corrélation entre la sévérité du PTI, la diminution des plaquettes et l'intensité de la MFI obtenue, a été mis en évidence par plusieurs auteurs, par ailleurs la MFI du contrôle négatif augmente également lorsque le taux de plaquette diminue [15].

Bien que la recherche directe ou indirecte des PAIG par cytométrie en flux, soit une technique très sensible avec une excellente valeur prédictive négative qui peut atteindre $100 \%$, elle reste très peu spécifique, en raison du taux élevé des PAIG retrouvé dans les thrombopénies nonimmunologiques, et même chez les sujets normaux. Cette mauvaise spécificité est inacceptable, et rend ce test inapproprié au diagnostic du PTI, à moins qu'il soit associé à d'autres techniques spécifiques comme la MAIPA ou l'ELISA [4, 13-15], ou bien d'autres modifications et améliorations soient apportées pour le rendre plus spécifique.

\section{QUANTIFICATION DES PAIG PAR CMF DIRECT}

Une quantification des immunoglobulines associées à la surface des plaquettes, peut améliorer la sensibilité et la spécificité de la recherche des PAIG par CMF. La quantification permet également le suivi et l'évaluation de la réponse des patients PTI aux traitements.

La quantification des PAIG, par méthode direct d'immunofluorescence par CMF, est réalisée à l'aide d'un étalonnage effectuée par des billes synthétiques marquées par une quantité connue d'immunoglobines couplées à l'FITC [16]. Une série de dilution de l'Ac est réalisée, la quantité des anticorps de surface sur les billes est mise en abscisse de la courbe d'étalonnage, et la MFI obtenue en ordonnée. Cette technique a permis d'augmenter la spécificité du test, qui était d'environ 30 à $40 \%$ avec la recherche conventionnelle des PAIG par cyrtométrie en flux, jusqu'à $66 \%$ avec la quantification par les billes fluorescentes, avec une sensibilité toujours excellente à $94 \%$ [16].

Une autre technique de quantification proposée par Christopoulos et al [10], consiste à utiliser des plaquettes à la place des billes. Ces plaquettes sont préalablement sensibilisées par une quantité connue d'un anticorps antiplaquettaire. Une courbe d'étalonnage est ensuite réalisée de la même façon qu'avec les billes.

Il existe plusieurs différences entre la fluorescence obtenue à partir des billes marquées et celle des cellules vivantes. Ceci est en rapport avec les différences en matière de la taille, la forme, la complexité intérieur et le signale d'autofluorescence.

La technique de quantification par étalonnage avec des billes a montré que le taux des Ig de surface, chez les patients PTI était à plus de 10000/plaquettes [16]. Par ailleurs l'étalonnage à l'aide des plaquettes vivantes sensibilisées [10] trouve une moyenne de 11535/plaquettes. 
Tableau 1 : Principales caractéristiques de certaines études de recherche des PAIG par cytométrie en flux.

\begin{tabular}{|c|c|c|c|c|c|c|}
\hline Auteur & $\begin{array}{l}\text { Fixation } \\
\text { des } \\
\text { plaquettes }\end{array}$ & Anticorps & Contrôle & $\begin{array}{l}\text { Expression } \\
\text { des résultats }\end{array}$ & Sensibilité & Spécificité \\
\hline Corash [21] & $\begin{array}{l}\text { PFH 1\% } \\
5 \mathrm{~min}\end{array}$ & $\begin{array}{l}\text { Biotine goat anti- } \\
\text { IgG humaine }\end{array}$ & Non & MFI & $93,8 \%$ & $94,5 \%$ \\
\hline $\begin{array}{l}\text { Rosenfeld [13] } \\
44 \text { PTI }\end{array}$ & Non & $\begin{array}{l}\text { FITC goat anti-IgG } \\
\text { humaine }\end{array}$ & Sheep anti-mouse & $\begin{array}{l}\% \text { des plaquettes positives } \\
\text { (moyenne }+3 \mathrm{SD} \text { ) }\end{array}$ & $92 \%$ & $81,8 \%$ \\
\hline $\begin{array}{l}\text { Christopoulos } \\
\text { [10] } \\
16 \text { PTI }\end{array}$ & Non & $\begin{array}{l}\text { FITC goat anti-IgG } \\
\text { humaine }\end{array}$ & $\begin{array}{l}\text { Non immune goat } \\
\text { IgG }\end{array}$ & $\begin{array}{l}\text { MFIgoatIgG/MFI contrôle = } \\
\text { RFI (moyenne RFI + 2SD) }\end{array}$ & $75 \%$ & $77,8 \%$ \\
\hline $\begin{array}{l}\text { Tazzari }[11] \\
54 \text { PTI }\end{array}$ & $\begin{array}{l}\text { PFH } 1 \%, \\
5 \mathrm{~min}\end{array}$ & $\begin{array}{l}\text { FITC goat anti-IgG } \\
\text { humaine }\end{array}$ & $\begin{array}{l}\text { Pttenormales + goat } \\
\text { anti-IgG humaine }\end{array}$ & MFI & $85 \%$ & $100 \%$ \\
\hline $\begin{array}{l}\text { Romero- } \\
\text { Guzman [8] } \\
62 \text { PTI }\end{array}$ & $\begin{array}{l}\text { PFH 2\%, } \\
10 \mathrm{~min}\end{array}$ & $\begin{array}{l}\text { FITC rabit anti-IgG, } \\
\text { IgM humaines }\end{array}$ & $\begin{array}{l}\text { FITC Goat anti- } \\
\text { mouse }\end{array}$ & $\begin{array}{l}\% \text { des plaquettes positives } \\
(\text { Moyenne }+2 \mathrm{SD})\end{array}$ & $90,3 \%$ & $39,3 \%$ \\
\hline $\begin{array}{l}\text { Lazarchik [15] } \\
8 \text { PTI }\end{array}$ & Non & $\begin{array}{l}\text { FITC goat anti-IgG } \\
\text { humaine }\end{array}$ & Non & $\begin{array}{l}\text { MFI ptte marquées /MFI ptte } \\
\text { non marquées }\end{array}$ & $63 \%$ & $60 \%$ \\
\hline $\begin{array}{l}\text { Campos } \\
\text { Guerra [12] } \\
8 \text { PTI }\end{array}$ & - & - & - & - & $76,9 \%$ & $83,3 \%$ \\
\hline $\begin{array}{l}\text { Huh }[14] \\
59 \text { PTI }\end{array}$ & Non & $\begin{array}{l}\text { FITC rabit anti-IgG } \\
\text { IgA IgM humaines }\end{array}$ & FITC mouse IgG & $\begin{array}{l}\% \text { des plaquettes positives } \\
\text { analyse par courbe ROC }\end{array}$ & $74 \%$ & $78 \%$ \\
\hline $\begin{array}{l}\text { Nishioka [9] } \\
23 \text { PTI }\end{array}$ & Non & $\begin{array}{l}\text { FITC anti total Ig, } \\
\text { IgG, IgM, IgA. }\end{array}$ & FITC anti-mouse IgG & $\begin{array}{l}\% \text { de positivité, au-delà du } \\
\text { signal du contrôle nég }\end{array}$ & $\begin{array}{c}\text { Total Ig } \\
95 \% \\
\text { Anti IgG } \\
69 \%\end{array}$ & / \\
\hline $\begin{array}{l}\text { Nielsen [6] } \\
88 \text { PTI }\end{array}$ & Non & FITC anti IgG, IgM & Non & MFI / courbe ROC & $74 \%$ & / \\
\hline $\begin{array}{l}\text { Hezard }[22] \\
73 \text { PTI }\end{array}$ & Non & $\begin{array}{l}\text { FITC goat anti IgG, } \\
\text { IgM, IgA }\end{array}$ & Non & MFI / courbe ROC & $100 \%$ & $57 \%$ \\
\hline $\begin{array}{l}\text { Hamidpour } \\
\text { [23] } \\
38 \text { PTI }\end{array}$ & Non & FITC anti IgG & Non & Moyenne MFI + 3SD & $62 \%$ & / \\
\hline $\begin{array}{l}\text { Stockelberg } \\
{[4]} \\
65 \text { PTI }\end{array}$ & Non & $\begin{array}{l}\text { FITC anti IgG, IgM, } \\
\text { IgA }\end{array}$ & Non & Moyenne MFI + 3SD & $45 \%$ & / \\
\hline
\end{tabular}

PFH : Paraformaldéhyde. MFI : Intensité Moyenne de Fluorescence. FITC : Isothiocyanate de fluorescéine. ROC : Receiver Operating Characteristic.

\section{IDENTIFICATION DES AUTO-ANTICORPS ANTI-GP PLAQUETTAIRES PAR CMF AVEC MICROBILLES (FCIA)}

FCIA (flow cytometric immuno-bead-assay), est un test développé récemment, il associe la rapidité, la reproductibilité, la précision et la sensibilité de la recherche des PAIG par CMF, avec la spécificité de la MAIPA, par la possibilité d'identification des GP plaquettaires cibles des auto-anticorps.

Le principe de la FCIA est basé sur l'utilisation des microbilles en polystyrène sensibilisées par des anticorps anti-GP plaquettaires. Les anticorps fixés sur les microbilles sont l'anti-GP : IX (SZ1), Ib (SZ2), IIIa (SZ21), IIb (SZ22) et Pselectine (SZ51). La recherche est effectuée soit sur les plaquettes du patient pour l'épreuve directe, ou bien en utilisant des plaquettes normales, sensibilisées par le sérum du patient, pour l'épreuve indirecte.
Les plaquettes lavées et sensibilisée par des anti-GP à leurs surfaces, sont solubilisées par le Triton X-100, une forte centrifugation ensuite 3000 à $10000 \mathrm{~g}$, permet de récupérer le lysat plaquettaire. Les billes marquées par les anti-GP sont incubées avec le lysat plaquettaire. Après le lavage, la révélation est faite par un FITC mouse anti-human-IgG. L'intensité de la fluorescence MFI est mesurée et comparée à celle de la population témoins (Figure1).

Une modification de la technique FCIA, est apportée afin de permettre en plus de l'identification des GP cibles, une quantification des ces anticorps. L'utilisation de microbilles possédant plusieurs niveaux de fluorescence (5 à 8), permet le traçage d'une courbe d'étalonnage (concentration IgG vs MFI) (tableau 2) [5,17-20].

La FCIA est une technique d'identification et quantification des auto-anticorps anti glycoprotéines plaquettaires. Bien que la majorité de ces anticorps sont dirigés contre la GPIbIX 


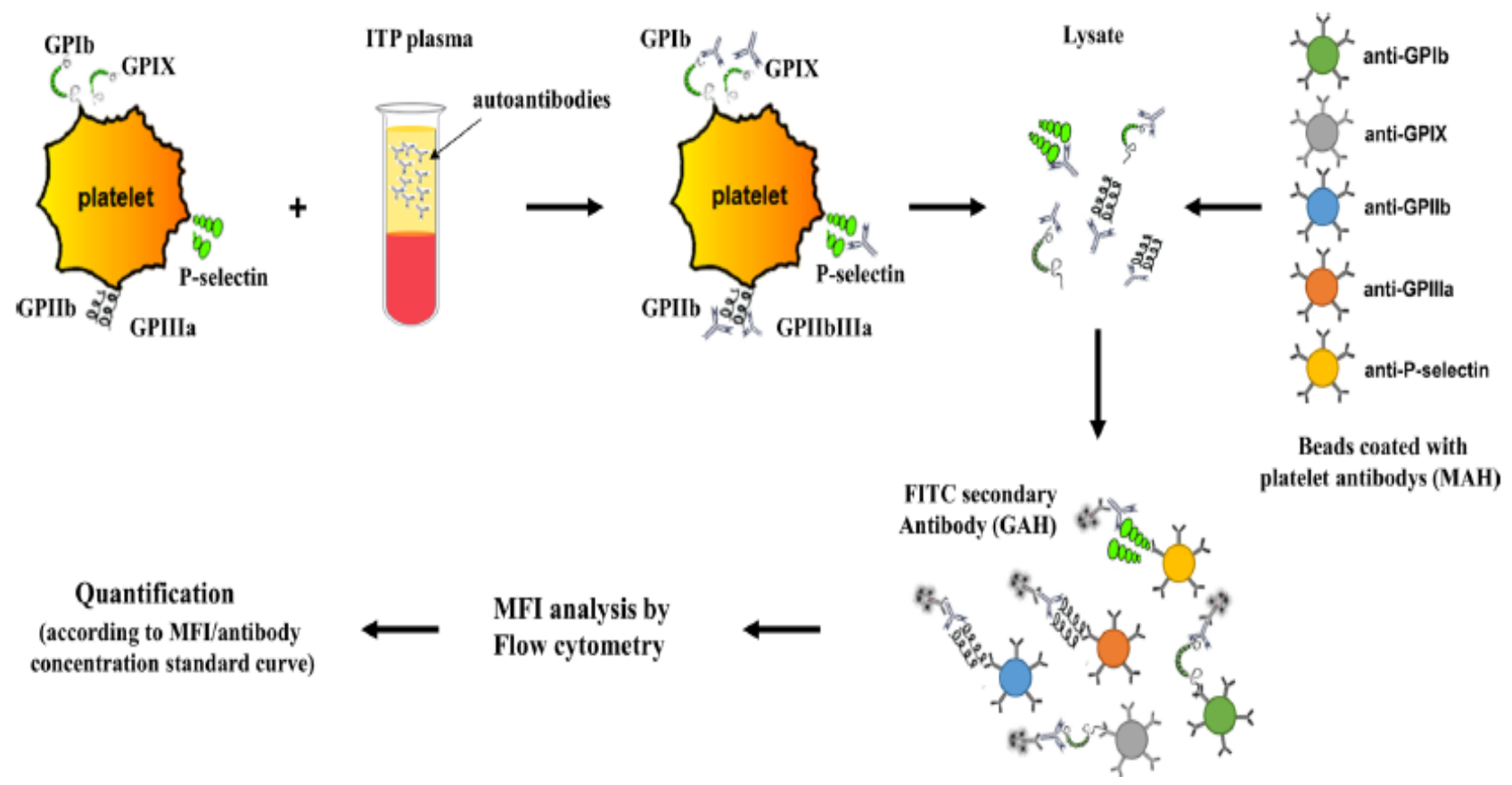

Figure 1. Principe de la FCIA selon Zhai et al [18]

Tableau 2. Principales caractéristiques de certaines études de FCIA.

\begin{tabular}{|c|c|c|c|c|c|c|}
\hline Auteur & Microbilles & $\begin{array}{l}\text { Anticorps } \\
\text { couplés aux } \\
\text { billes }\end{array}$ & $\begin{array}{l}\text { Anticorps de } \\
\text { détection }\end{array}$ & Contrôle & Sensibilité & Spécificité \\
\hline $\begin{array}{l}\text { Zhai [18] } \\
250 \text { PTI }\end{array}$ & $\begin{array}{l}04 \text { um } \\
08 \text { niveaux de } \\
\text { fluorescence }\end{array}$ & $\begin{array}{l}\text { GPIX, GPIb, GPIIb, } \\
\text { GPIIIa, P- } \\
\text { sélectine }\end{array}$ & $\begin{array}{l}\text { FITC Goat anti- } \\
\text { humain IgG }\end{array}$ & $\begin{array}{l}\text { FITC goat anti-mouse } \\
\text { IgG }\end{array}$ & $73,13 \%$ & $81,98 \%$ \\
\hline $\begin{array}{l}\mathrm{He}[5] \\
\text { 50 PTI }\end{array}$ & $\begin{array}{l}04 \text { um } \\
08 \text { niveaux de } \\
\text { fluorescence }\end{array}$ & $\begin{array}{l}\text { GPIX, GPIb, GPIIb, } \\
\text { GPIIIa, P- } \\
\text { sélectine }\end{array}$ & $\begin{array}{l}\text { FITC Goat anti- } \\
\text { humain IgG }\end{array}$ & $\begin{array}{l}\text { FITC goat anti-mouse } \\
\text { IgG }\end{array}$ & $96 \%$ & $80,9 \%$ \\
\hline $\begin{array}{l}\text { Zhao [17] } \\
\text { 71 PTI }\end{array}$ & $\begin{array}{l}04 \text { um } \\
\text { Plusieurs intensités }\end{array}$ & $\begin{array}{l}\text { GPIX, GPIb, GPIIb, } \\
\text { GPIIIa, P- } \\
\text { sélectine }\end{array}$ & $\begin{array}{l}\text { FITC Goat anti- } \\
\text { humain IgG }\end{array}$ & $\begin{array}{l}\text { FITC goat anti-mouse } \\
\text { IgG }\end{array}$ & $78,3 \%$ & $75 \%$ \\
\hline $\begin{array}{l}\text { Liu }[19] \\
123 \text { PTI }\end{array}$ & 15,3 um & $\begin{array}{l}\text { GPIIbIIIa (CD41) } \\
\text { GPIbIX (CD42) }\end{array}$ & $\begin{array}{l}\text { Polyclonal PE } \\
\text { Goatanti- } \\
\text { humainIgG }\end{array}$ & - & $73 \%$ & $95 \%$ \\
\hline $\begin{array}{l}\text { Tomer [20] } \\
\text { 62 PTI }\end{array}$ & $20 \mathrm{um}$ & GPIIbIIIa & $\begin{array}{l}\text { FITC Goat anti- } \\
\text { humain IgG }\end{array}$ & $\begin{array}{l}\text { FITC goat anti-mouse } \\
\text { IgG }\end{array}$ & $66 \%$ & $93 \%$ \\
\hline
\end{tabular}

et la GPIIbIIIa, l'association de 5 anticorps différents antiGPIb, IX, IIb, IIIa et P-sélectine, augmente la spécificité du test (entre 75 et 81\%). Néanmoins l'utilisation des deux anticorps anti CD41 et anti CD42, semble être suffisante aussi (une sensibilité à $73 \%$ et une spécificité à 95\%) [19], par contre l'utilisation du CD41 seul, diminue la sensibilité du test jusqu'à $66 \%$.

La FCIA a montrée qu'elle surpasse la MAIPA et toutes les autres techniques, dans la recherche, l'identification et la quantification des anticorps antiplaquettaires. Elle offre le meilleur compromis entre sensibilité et spécificité en comparaison avec la MAIPA $[5, \mathbf{1 7}, \mathbf{1 8}]$.

Déclaration d'intérêts: les auteurs ne déclarent aucun conflit d'intérêt en rapport avec cet article.

\section{RÉFÉRENCES}

1. Neunert C, Lim W, Crowther M, Cohen A, Solberg L Jr, Crowther MA; American Society of Hematology. The American Society of Hematology 2011 evidence-based practice guideline for immune thrombocytopenia. Blood. 2011 Apr 21;117(16):4190-207. doi: 10.1182/blood-2010-08302984. Epub 2011 Feb 16. PMID: 21325604.

2. Moulis G, Lapeyre-Mestre M, Adoue D, Sailler L. Épidémiologie et pharmacoépidémiologie du purpura thrombopénique immunologique [Epidemiology and pharmacoepidemiology of immune thrombocytopenia]. Rev Med Interne. 2017 Jul;38(7):444-449. French. doi: 10.1016/j.revmed.2016.12.016. Epub 2017 Jan 26. PMID: 28131440.

3. Provan D. Characteristics of immune thrombocytopenic purpura: a guide for clinical practice. Eur J Haematol Suppl. 2009 Mar;(71):8-12. doi: 10.1111/j.1600-0609.2008.01207.x. PMID: 19200302.

4. Stockelberg D, Hou M, Jacobsson S, Kutti J, Wadenvik H. Detection of platelet antibodies in chronic idiopathic thrombocytopenic purpura (ITP). A comparative study using flow cytometry, a whole platelet ELISA, and an antigen capture ELISA. Eur J Haematol. 1996 Jan-Feb;56(1-2):72-7. doi: 10.1111/j.1600-0609.1996.tb00298.x. PMID: 8599998. 
5. He Y, Zhao YX, Zhu MQ, Wu Q, Ruan CG. Detection of autoantibodies against platelet glycoproteins in patients with immune thrombocytopenic purpura by flow cytometric immunobead array. Clin Chim Acta. 2013 Jan 16;415:176-80. doi: 10.1016/j.cca.2012.10.035. Epub 2012 Oct 25. PMID: 23103637.

6. Nielsen $\mathrm{OH}$, Tuckuviene R, Nielsen KR, Rosthøj S. Flow cytometric measurement of platelet-associated immunoglobulin in children with newly diagnosed Immune Thrombocytopenia. Eur J Haematol. 2016 Apr;96(4):397-403. doi: 10.1111/ejh.12605. Epub 2015 Jun 30. PMID: 26111053.

7. George JN, Woolf SH, Raskob GE, Wasser JS, Aledort LM, Ballem PJ, Blanchette VS, Bussel JB, Cines DB, Kelton JG, Lichtin AE, McMillan R, Okerbloom JA, Regan DH, Warrier I. Idiopathic thrombocytopenic purpura: a practice guideline developed by explicit methods for the American Society of Hematology. Blood. 1996 Jul 1;88(1):3-40. PMID: 8704187.

8. Romero-Guzmán LT, López-Karpovitch X, Paredes R, Barrales-Benitez O, Piedras J. Detection of platelet-associated immunoglobulins by flow cytometry for the diagnosis of immune thrombocytopenia: a prospective study and critical review. Haematologica. 2000 Jun;85(6):627-31. PMID: 10878748.

9. Nishioka $T$, Yamane $T$, Takubo $T$, Ohta $K$, Park $K$, Hino $M$. Detection of various platelet-associated immunoglobulins by flow cytometry in idiopathic thrombocytopenic purpura. Cytometry B Clin Cytom. 2005 Nov;68(1):37-42. doi: 10.1002/cyto.b.20067. PMID: 16184616.

10. Christopoulos CG, Kelsey HC, Machin SJ. A flow-cytometric approach to quantitative estimation of platelet surface immunoglobulin G. Vox Sang. 1993;64(2):106-15. doi: 10.1111/j.1423-0410.1993.tb02527.x. PMID: 8456554 .

11. Tazzari PL, Ricci F, Vianelli N, Tassi C, Belletti D, Pierri I, Gugliotta L, Gobbi $\mathrm{M}$, Conte R. Detection of platelet-associated antibodies by flow cytometry in hematological autoimmune disorders. Ann Hematol. 1995 May;70(5):267-72. doi: 10.1007/BF01784046. PMID: 7599288.

12. Guerra JC, Kanayama RH, Nozawa ST, loshida MR, Takiri IY, Lazaro RJ, Hamerschlak N, Rosenfeld LG, Guerra CC, Bacal NS. Thrombocytopenia: diagnosis with flow cytometry and antiplatelet antibodies. Einstein (Sao Paulo). 2011 Jun;9(2):130-4. English, Portuguese. doi: 10.1590/S1679. 45082011AO1846. PMID: 26760804.

13. Rosenfeld CS, Nichols G, Bodensteiner DC. Flow cytometric measurement of antiplatelet antibodies. Am J Clin Pathol. 1987 Apr;87(4):518-22. doi: 10.1093/ajcp/87.4.518. PMID: 3103420.
14. Huh HJ, Park CJ, Kim SW, Han SH, Jang S, Chi HS. Flow cytometric detection of platelet-associated immunoglobulin in patients with immune thrombocytopenic purpura and nonimmune thrombocytopenia. Ann Clin Lab Sci. 2009 Summer;39(3):283-8. PMID: 19667413.

15. Lazarchick J, Hall SA. Platelet-associated IgG assay using flow cytometric analysis. J Immunol Methods. 1986 Mar 13;87(2):257-65. doi: 10.1016/0022-1759(86)90540-5. PMID: 3512726.

16. Hagenström $\mathrm{H}$, Schlenke $\mathrm{P}$, Hennig $\mathrm{H}$, Kirchner $\mathrm{H}$, Klüter $\mathrm{H}$. Quantification of platelet-associated IgG for differential diagnosis of patients with thrombocytopenia. Thromb Haemost. 2000 Nov;84(5):779-83. PMID: 11127855.

17. Zhao $\mathrm{Y}$, Zhu $\mathrm{M}$, Jiang $\mathrm{M}$, Zuo B, Wu Q, Ruan $\mathrm{C}$, He $\mathrm{Y}$. An improved flow cytometric immunobead array to detect autoantibodies in plasma from patients with immune thrombocytopenic purpura. Clin Chim Acta. 2015 Jan 1;438:396-400. doi: 10.1016/j.cca.2014.09.018. Epub 2014 Sep 28. PMID: 25269089.

18. Zhai J, Ding M, Yang T, Zuo B, Weng Z, Zhao Y, He J, Wu Q, Ruan C, He Y. Flow cytometric immunobead assay for quantitative detection of platelet autoantibodies in immune thrombocytopenia patients. J Transl Med. 2017 Oct 23;15(1):214. doi: 10.1186/s12967-017-1317-2. PMID: 29061180; PMCID: PMC5654144.

19. Liu XG, Li JL, Qin P, Ren J, Ma SH, Sun L, Shi Y, Ji XB, Zhu YY, Ma DX, Guo CS, Du X, Hou M, Peng J. Determination of platelet-bound glycoproteinspecific autoantibodies by flow cytometric immunobead assay in primary immune thrombocytopenia. Eur J Haematol. 2011 Apr;86(4):339-46. doi: 10.1111/j.1600-0609.2010.01572.x. Epub 2011 Feb 18. PMID: 21198863.

20. Tomer A, Koziol J, McMillan R. Autoimmune thrombocytopenia: flow cytometric determination of platelet-associated autoantibodies against platelet-specific receptors. J Thromb Haemost. 2005 Jan;3(1):74-8. doi: 10.1111/j.1538-7836.2004.01052.x. PMID: 15634268.

21. CORASH, LAURENCE et RHEINSCHMIDT, MARGARET. Detection of platelet antibodies with a fluorescence activated flow cytometric technique. Rose, Friedman, Fahey, eds: Manual of Clinical Laboratory Immunology. Washington: American Society for Microbiology, 1986, p. 254-7.

22. Hézard N, Simon G, Macé C, Jallu V, Kaplan C, Nguyen P. Is flow cytometry accurate enough to screen platelet autoantibodies? Transfusion. 2008 Mar;48(3):513-8. doi: 10.1111/j.1537-2995.2007.01556.x. Epub 2007 Dec 7. PMID: 18067500.

23. Hamidpour M, Khalili G, Tajic N, Shamsian BB, Hamidpour R. Comparative of three methods (ELIZA, MAIPA and flow cytometry) to determine antiplatelet antibody in children with ITP. Am J Blood Res. 2014 Dec 15;4(2):86-92. PMID: 25755908; PMCID: PMC4348796.
Cet article a été publié dans le « Batna Journal of Medical Sciences » BJMS, l’organe officiel de « l'association de la Recherche Pharmaceutique - Batna »

Le contenu de la Revue est ouvert « Open Access » et permet au lecteur de télécharger, d'utiliser le contenu dans un but personnel ou d'enseignement, sans demander l'autorisation de l'éditeur/auteur.

Avantages à publier dans BJMS :

- Open access : une fois publié, votre article est disponible gratuitement au téléchargement

- Soumission gratuite : pas de frais de soumission, contrairement à la plupart des revues « Open Access »

- Possibilité de publier dans 3 langues : français, anglais, arabe

- Qualité de la relecture : des relecteurs/reviewers indépendants géographiquement, respectant l'anonymat, pour garantir la neutralité et la qualité des manuscrits.

Pour plus d'informations, contacter BatnaJMS@gmail.com ou connectez-vous sur le site de la revue : www.batnajms.net
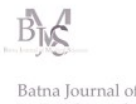

Medical Sciences

6 